\title{
BMJ Open Lung protective ventilation in patients undergoing major surgery: a systematic review protocol
}

\author{
Zhongheng Zhang, ${ }^{1}$ Xiaoyun $\mathrm{Hu},{ }^{2}$ Xia Zhang, ${ }^{1}$ Xiuqi Zhu, ${ }^{1}$ Li Zhu, ${ }^{1}$ Liqian Chen, ${ }^{1}$ \\ Jiaping Huai, ${ }^{1}$ Bin $\mathrm{Du},{ }^{2}$ for the China Critical Care Clinical Trials Group (CCCCTG)
}

To cite: Zhang Z, Hu X, Zhang $X$, et al. Lung protective ventilation in patients undergoing major surgery: a systematic review protocol. BMJ Open 2014;4 e004542. doi:10.1136/ bmjopen-2013-004542

- Prepublication history and additional material for this paper is available online. To view these files please visit the journal online (http://dx.doi.org/10.1136/ bmjopen-2013-004542).

Received 26 November 2013 Revised 2 February 2014 Accepted 21 February 2014

\section{CrossMark}

\footnotetext{
${ }^{1}$ Department of Critical Care Medicine, Jinhua Municipal Central Hospital, Jinhua Hospital of Zhejiang University, Zhejiang, People's Republic of China ${ }^{2}$ Medical ICU, Peking Union Medical College Hospital, Beijing, People's Republic of China
}

Correspondence to Dr Bin Du; dubin98@gmail.com

\begin{abstract}
Introduction: There is growing interest in the use of low tidal volume ventilation in patients undergoing general anaesthesia. However, its potential benefit has long been debated and conflicting results have been reported. We describe here the protocol of a systematic review and meta-analysis for investigating the beneficial effects of low tidal volume ventilation in patients undergoing general anaesthesia.
\end{abstract}

Methods and analysis: Data sources include PubMed, Scopus, Embase and EBSCO. Patients undergoing general anaesthesia will be included irrespective of type of surgery. The intervention is low tidal volume ventilation or protective ventilation, and the control is conventional ventilation. The quality of included trials will be assessed by using Delphi consensus. Outcomes include new onset lung injury, atelectasis, arrhythmia, levels of inflammatory biomarkers, arterial oxygenation, partial pressure of carbon dioxide and alveolar-arterial oxygen gradient. Conventional approaches for meta-analysis will be used, and heterogeneity will be investigated by using subgroup analysis and meta-regression if appropriate. The Bayesian method will be used for the synthesis of binary outcome data.

Ethics and dissemination: The systematic review was approved by the ethics committee of Jinhua hospital of Zhejiang university and will be published in a peer-reviewed journal and will be disseminated electronically and in print.

Registration details: The study protocol has been registered in PROSPERO (http://www.crd.york.ac.uk/ PROSPER0/) under registration number CRD42013006416.

\section{INTRODUCTION}

It is estimated that 234.2 million cases $(95 \%$ CI 187.2 to 281.2) of major surgery were carried out worldwide in 2004, corresponding to about one operation for every 25 people. ${ }^{1}$ Postoperative pulmonary complications associated with general anaesthesia are a major cause of perioperative mortality and morbidity. $^{2-4}$ The induction of general anaesthesia may cause a significant decrease in lung volume and atelectasis, which in turn results in impairment in gas exchange and pulmonary mechanics. 56

A large body of evidence from animal experiments has demonstrated that mechanical ventilation can initiate lung injury, even in healthy lungs. ${ }^{7-9}$ Serpa Neto and colleagues, in a meta-analysis of 20 papers involving 2822 patients without acute respiratory distress syndrome (ARDS), found that protective ventilation with lower tidal volumes was associated with a decrease in lung injury (risk ratio (RR) $0.33,95 \%$ CI 0.23 to $0.47 ; \mathrm{p}<0.001)$ and mortality (RR $0.64,95 \%$ CI 0.46 to 0.89 ; $\mathrm{p}=0.007) .{ }^{10}$ However, five observational studies included in this meta-analysis accounted for approximately $85 \%$ of both the number of patients and events in the primary analysis of lung injury prevention. ${ }^{11}$ Furthermore, the effect of positive end-expiratory pressure (PEEP) was not explored in this meta-analysis, as PEEP levels were comparable between the study and control arms in some studies but significantly different in other studies. As a result, the use of lung protective ventilation in patients undergoing major surgery still remains controversial. ${ }^{11} 12$

Since 2009, a number of prospective randomised trials have been performed to investigate the efficacy of lung protective ventilation in patients without ARDS. ${ }^{13-21}$ We describe here the protocol of a systematic review to investigate whether lung protective ventilation is beneficial in patients undergoing major surgery. This systematic review has been registered with PROSPERO (the NIHR International Prospective Register of Systematic Reviews) under registration number CRD42013006416.

\section{METHODS}

Search methods for identifying studies

Electronic searches

We will search the databases PubMed, Scopus, EBSCO and Embase from inception 
to November 2013. There will be no language restrictions in the electronic search for trials.

\section{Search terms/search strategy}

The search strategy has been developed for PubMed and consists of terms related to surgery and lung protective ventilation (table 1). The PubMed strategy will be adapted for the other databases.

\section{Study inclusion criteria}

Studies to be included

Studies meeting the following criteria will be included: (1) the study population should consist of patients undergoing mechanical ventilation after induction of general anaesthesia, and can include adults and/or children; (2) the intervention should be lung protective ventilation while the control arm uses the conventional ventilation strategy. Exclusion criteria include: (1) nonexperimental studies (observational studies, case-control studies or secondary analysis of data from randomised controlled trials (RCT)); (2) animal studies; and (3) articles such as reviews, comments and letters.

\section{Intervention}

Lung protective ventilation, that is, mechanical ventilation with low tidal volumes with or without the differential use of PEEP and/or recruitment manoeuvres. Low tidal volume is defined as $\leq 8 \mathrm{~mL} / \mathrm{kg}$ of predicted body weight.

\section{Comparison}

Ventilation strategy using the conventional tidal volume of $\geq 8 \mathrm{~mL} / \mathrm{kg}$ of predicted body weight as the control.

\section{Outcome}

Primary outcomes are incidence of acute lung injury (ALI) and ARDS. ALI and ARDS are defined according to the Berlin definition or the American-European Consensus Conference (AECC) definition. ${ }^{22}{ }^{23}$ ARDS is defined as the acute onset of hypoxaemia (the ratio of partial pressure of arterial oxygen to fraction of inspired oxygen $\left.\left(\mathrm{PaO}_{2} / \mathrm{FiO}_{2}\right) \leq 200 \mathrm{~mm} \mathrm{Hg}\right)$, with bilateral infiltrates on frontal chest X-ray, in the absence of left atrial hypertension (determined by pulmonary capillary wedge pressure $<18 \mathrm{~mm} \mathrm{Hg}$ ). ALI was defined by using the same variables but with a less stringent criterion for

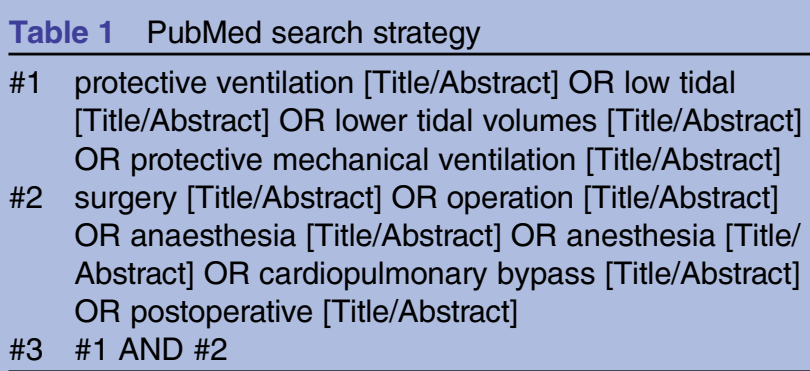

hypoxaemia $\left(\mathrm{PaO}_{2} / \mathrm{FiO}_{2} \leq 300 \mathrm{~mm} \mathrm{Hg}\right)$. The Berlin definition states that: (1) onset should be within 1 week of a known clinical insult or new/worsening respiratory symptoms; (2) chest imaging should show bilateral opacities that cannot be fully explained by effusions, lobar/ lung collapse or nodules; and (3) respiratory failure not fully explained by cardiac failure or fluid overload should be present; objective assessment may be needed to exclude hydrostatic oedema if no risk factor is present. ARDS is divided into three mutually exclusive categories of mild $\left(200<\mathrm{PaO}_{2} / \mathrm{FiO}_{2} \leq 300\right.$ with PEEP or continuous positive airway pressure $(\mathrm{CPAP}) \geq 5 \mathrm{~cm} \mathrm{H}_{2} \mathrm{O}$ ), moderate $\left(100<\mathrm{PaO}_{2} / \mathrm{FiO}_{2} \leq 200\right.$ with $\left.\mathrm{PEEP} \geq 5 \mathrm{~cm} \mathrm{H} \mathrm{H}_{2} \mathrm{O}\right)$ and severe $\left(\mathrm{PaO}_{2} / \mathrm{FiO}_{2} \leq 100\right.$ with PEEP $\left.\geq 5 \mathrm{~cm} \mathrm{H} \mathrm{H}_{2} \mathrm{O}\right)$.

Clinical outcomes are intensive care unit length of stay (LOS), hospital LOS and mortality. These definitions may differ across component studies, and we will retrieve the data according to the following definitions:

Oxygenation: arterial $\mathrm{pH}$ value, $\mathrm{PaCO}_{2}, \mathrm{PaO}_{2}, \mathrm{PaO}_{2} /$ $\mathrm{FiO}_{2}$, alveolar-arterial oxygen gradient

Complications: atelectasis, new onset arrhythmia Inflammatory markers in serum and bronchoalveolar lavage fluid: interleukin-6 (IL-6), interleukin-8 (IL-8)

Type of studies

We will include any RCT.

\section{Data collection and analysis}

\section{Selection of studies}

The initial search and selection of potentially relevant studies by inspection of titles and abstracts will be conducted by three review authors. XHu will search Embase, LChen and XZhang will search PubMed, XZhu and JHuai will search EBSCO, and XZhu and LChen will search Scopus. All these review authors will conduct searches independently. Any disparities will be resolved by a third opinion. The full text of eligible studies obtained in the first step will be reviewed. Any mistakes in the first step or uncertainty about the inclusion or exclusion of a study will be resolved by careful review of the full text article. Corresponding authors will be contacted if further information is needed.

\section{Data extraction and management}

A standard form for data collection will be developed. Data will be gathered at study level. The information collected on component studies will include study population, setting, type of surgery, description of surgery (eg, elective vs urgent), blood transfusion, fluid balance, mean or median age, sex (the proportion of male patients), tidal volume settings in the study and control arms, use of recruitment manoeuvres, and PEEP level. If a study reports age separately for the study and control arms, the weighted mean difference will be reported in our analysis. Three investigators (XZ, LC, XH) will independently extract data from component studies and any disagreement will be settled by consulting a fourth investigator $(\mathrm{ZZ})$. 
Assessment of the quality of included studies

Quality assessment of all included randomised controlled trials will be based on Delphi consensus (table 2), which covers the following eight aspects: sequence generation, allocation concealment, blinding, eligibility criteria, baseline characteristics, use of point estimates and variability, intention-to-treat analysis, and sample size calculation. ${ }^{24}$

\section{Statistical analysis and data synthesis}

Two categories of data will be reported as study endpoints in the present analysis: binary outcome measuring the risk of specific events (such as the occurrence of ALI, new onset arrhythmia and death) and continuous outcomes (such as the level of inflammatory markers, LOS, $\mathrm{PaO}_{2} / \mathrm{FiO}_{2}$ and $\mathrm{PaCO}_{2}$ ).
Report of effect size

The effect size of component studies will be pooled by using the inverse-variance method. ${ }^{25}$ Mantel-Haenszel methods will be used in situations where there are few events. For instance, because mortality is expected to be low in patients undergoing general anaesthesia, the Mantel-Haenszel method will be used for the synthesis of RR or OR for mortality. For continuous outcomes, we will use the mean difference as the effect size. However, because the types of inflammatory markers vary across studies, even if two studies report the same marker, the sample used can be different. We believe that it is more relevant to report whether the use of lung protective ventilation can reduce proinflammatory cytokines, while absolute values are not so important. Therefore, we will use standardised mean difference to

Table 2 Format for assessment of methodological quality adapted from Delphi consensus

Quality indicator
Sequence generation
Allocation concealment
Eligibility clearly described

Is the outcome assessor blind to the assignment?

Is the treatment provider blind to the assignment?

Is the patient blind to the assignment?

Are baseline characteristics comparable between the treatment and control arms?

Are point estimates and measures of variability presented for the primary outcome measures?

Is the sample size calculated?

Is intention-to-treat analysis employed?

\section{Description}

'Yes' if randomisation sequence number generation is described as 'random number table' or 'computer generated'. 'No' if randomisation is performed according to alternate admission date, odd or even number of patient ID. 'Unclear' if this is not specifically described in the text. 'Yes' if allocation sequence is concealed from those assigning participants to intervention groups until the moment of assignment. An opaque envelope is a typical method to achieve allocation concealment. 'No' if the investigator is aware of the assignment before assignment. 'Unclear' if the text does not give information on this item. 'Yes' if the inclusion and exclusion criteria are explicitly described. 'No' if the inclusion and exclusion criteria are vague or only a general description is provided.

'Yes' if the outcome assessor is unaware of the assignment of patients; outcome assessors are those who evaluate chest $\mathrm{X}$-ray for inflammatory biomarkers. 'No' if the outcome assessor is aware of the assignment. 'Unclear' if the study does not report information on this item.

Treatment provider includes anaesthesiologist and surgeon because both provide intervention directly to patients. 'Yes' if they are blind to the assignment of the participant. 'No' if the surgeon or anaesthesiologist is aware of the assignment. 'Unclear' if the study does not report information on this item.

'Yes' if the patient is blind to the treatment assignment. 'No' if the patient is not blind to the treatment assignment, for instance, the patient is told about the treatment assignment after operation. 'Unclear' if insufficient information is provided.

'Yes' if $>90 \%$ of investigated parameters are not statistically different between the treatment and control arms; component studies typically list the baseline characteristics (see table 1 of original paper). 'No' if otherwise. 'Unclear' if insufficient information is provided.

'Yes' if point estimates and measures of variability are presented for the primary outcome measures. The point estimates included median and mean, and variability includes SE and IQR. 'No' if these are not present. 'Unclear' if insufficient information is provided.

'Yes' if statistical power and $\alpha$ level are employed to calculate estimated sample size. 'No' if this is not described in the text. 'Yes' if the final analysis includes every subject who is randomised according to randomised treatment assignment. 'No' if the final analysis includes only those who have completed the study. 'Unclear' if insufficient information is provided. 
combine these results, in which the standard deviations are used to standardise the mean differences to a single scale.

\section{Assessment of heterogeneity}

Heterogeneity will be assessed by using statistic $\mathrm{I}^{2}$, where $\mathrm{I}^{2}=0 \%$ suggests no observed heterogeneity, and larger values indicate increasing heterogeneity. $\mathrm{I}^{2}$ will be calculated according to the equation $\mathrm{I}^{2}=100 \% \times(\mathrm{Q}-\mathrm{df}) / \mathrm{Q}$ where $\mathrm{Q}$ is the Cochran heterogeneity statistic. We predefine that $\mathrm{I}^{2}>50 \%$ indicates statistically significant heterogeneity. ${ }^{26} \mathrm{~A}$ random effect model will be used to synthesise the pooled effect size when there is significant heterogeneity; otherwise, a fixed effect model will be used.

\section{Subgroup analysis}

Subgroup analysis will be used to explore heterogeneity among component studies. We believe that differential use of tidal volume, PEEP and recruitment manoeuvre (open lung technique) between the study and control arms may confound outcome, and so we will perform subgroup analysis on these variables. Furthermore, patients undergoing urgent surgery may differ from those undergoing elective surgery in that they may have risk factors for ARDS (sepsis, shock, organ failure, multiple blood transfusions) and therefore have a different basal status compared with those undergoing scheduled major surgery. Subgroup analysis will also be performed on patients undergoing urgent versus elective surgery. Meta-regression analysis can be employed if the modifying covariates are continuous variables. In the meta-regression model, an outcome variable is predicted according to the values of one or more explanatory variables. ${ }^{27}$

\section{Sensitivity analysis}

Sensitivity analysis will be performed by excluding studies with poor methodological design in order to test the robustness of our results.

\section{Publication bias}

Publication bias will be assessed by using Begg's rank correlation test and Egger's regression test. ${ }^{28} \mathrm{~A}$ funnel plot will be used to visually assess the presence of publication bias. The OR is plotted on the $\mathrm{x}$-axis against its precision on the y-axis, and asymmetrical distribution of component studies may indicate potential publication bias. Begg's rank correlation test uses the Spearman rank correlation to investigate the relationship between the standardised effect size and sample size or variance in effect size. ${ }^{29}$ In Egger's regression test, the standard normal deviate (the OR divided by its SE) is regressed against the estimates precision. The intercept of the regression line provides an estimate of asymmetry-the larger its deviation from origin, the more significant the asymmetry. ${ }^{30}$

\section{Assessment of small-study effects}

Because the sample sizes of some component studies are expected to be relatively small, a small-study effect may be present. ${ }^{31}$ As a result, we plan to use the full Bayesian approach to address this issue. Bayesian methods provide a unified modelling framework which overcomes issues including those related to the appropriate treatment of small trials, with flexibility allowing for non-

Table 3 Script for running WinBUGS

\begin{tabular}{|c|c|}
\hline Random effects model & Fixed effect model \\
\hline 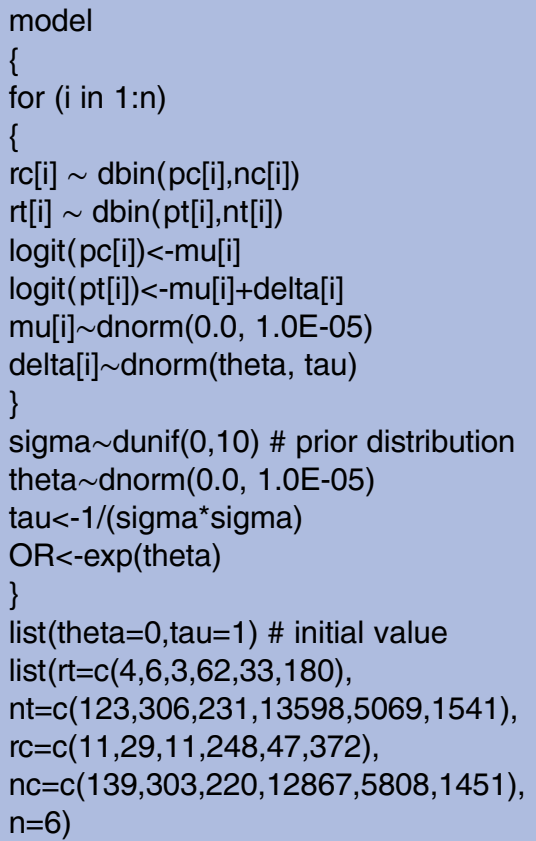 & $\begin{array}{l}\text { model } \\
\{ \\
\text { for }(i \text { in } 1: n) \\
\{ \\
\text { rc }[i] \sim d b i n(p c[i], n c[i]) \\
\text { rt[i] } \sim \operatorname{dbin}(p t[i], n t[i]) \\
\text { logit }(p c[i])<-m u[i] \\
\text { logit }(p t[i])<-m u[i]+\text { delta } \\
\text { mu[i] dnorm(0.0, 1.0E-05) } \\
\} \\
\text { delta dnorm(0.0, 1.0E-05) } \\
\text { OR<-exp(delta) } \\
\}\end{array}$ \\
\hline
\end{tabular}


normal distribution for random effects. ${ }^{32}$ The effect of lung protective ventilation compared with conventional intervention in pulmonary complications, new onset arrhythmia and other binary outcome variables will be assessed by Bayesian random-effect models using WinBUGS V.1.4. The code for the random effect model and the fixed effect model in WinBUGS is given in table 3. Figure 1 shows the WinBUGS flowchart.

\section{DISCUSSION}

Despite being the standard of care for the treatment of ARDS, ${ }^{33}$ it is not clear whether lung protective ventilation offers benefits in healthy lungs. General anaesthesia requiring mechanical ventilation is widely employed during surgery, when the lungs under mechanical ventilation are mostly healthy. The conventional ventilation strategy recommends the use of high tidal volume (10-15 mL/ $\mathrm{kg}$ predicted body weight) to prevent anaesthesia-induced hypoxaemia and atelectasis. On the other hand, the marked decrease in lung volume following laparotomy might, to some extent, be similar to the findings in patients with ARDS, where only $30-40 \%$ of alveoli are normally aerated due to alveolar collapse. ${ }^{34}$ Lung protective ventilation in this setting might be a rational strategy to prevent ventilator-induced lung injury.

A systematic review has been published by Serpa Neto and colleagues, ${ }^{10}$ suggesting that lung protective ventilation was associated with better clinical outcomes among patients without ARDS. However, there are major differences between the above study and our planned meta-analysis.

First, five out of 20 clinical trials included in Neto's study were observational studies, which might have significantly compromised the quality of the evidence, and should be regarded as hypothesis generating rather than hypothesis validating. ${ }^{35}$ In comparison, we will include only prospective randomised trials.

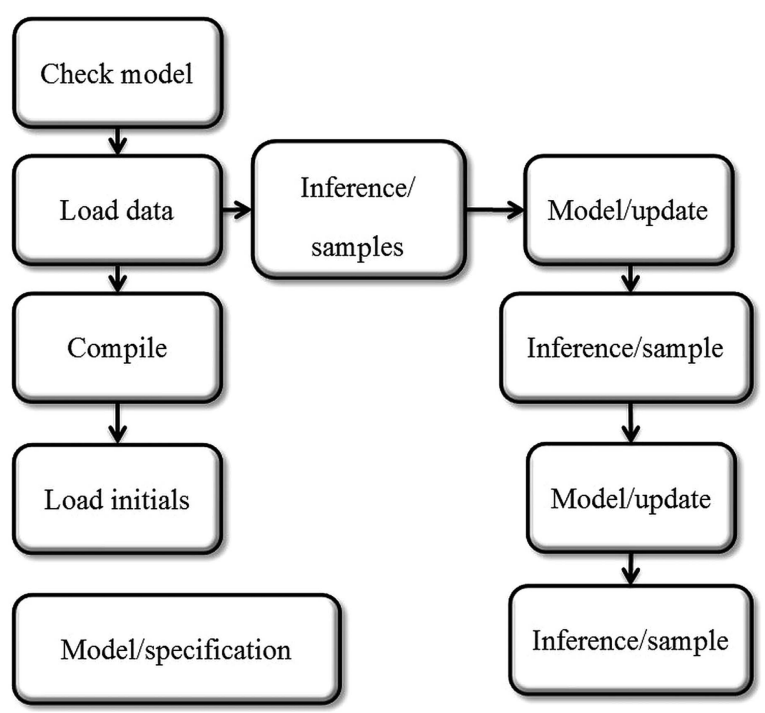

Figure 1 WinBUGS flow chart.
Second, medical patients without ARDS were also included in Neto's study. However, diagnosing ARDS is at times challenging. Among 255 patients who did not meet the clinical diagnostic criteria of ARDS, clinical autopsy found evidence of diffuse alveolar damage in 28 (11\%) patients. ${ }^{36}$ Interestingly, the difficulty of diagnosing of ARDS has not been improved by the Berlin ARDS definition, which had a sensitivity of $89 \%$ and specificity of only $63 \% .{ }^{37}$ The benefit of lung protective ventilation is therefore expected and self-explanatory. In our planned meta-analysis, we will only include patients undergoing major surgery in order to minimise the confounding effect of clinical diagnosis.

Last, but not the least, the sample sizes in component studies are relatively small, potentially giving rise to the so-called small-study effect. ${ }^{38}$ The common approach to meta-analysis is to assume that summary statistics have a normal likelihood, which however is usually not tenable in small trials. One approach to this problem is the use of the Bayesian method. In our analysis, we will employ the Bayesian method for both random-effect and fixed-effect models.

In conclusion, the current meta-analysis is aimed at investigating the beneficial effects of lung protective ventilation including low tidal volume with or without the use of PEEP and/or recruitment manoeuvre, in patients undergoing major surgery. We hope that the result of this meta-analysis will provide additional evidence on the practice of mechanical ventilation during the perioperative period in these patients.

Contributors BDu conceived the idea and designed the study; ZZhang drafted the manuscript; LZhu, LChen and XZhu collected the data and helped with statistical analysis; and LChen, XHu and JHuai helped to abstract data and review the manuscript.

\section{Competing interests None.}

Ethics approval The study was approved by ethics committee of Jinhua hospital of Zhejiang university.

Provenance and peer review Not commissioned; externally peer reviewed.

Open Access This is an Open Access article distributed in accordance with the Creative Commons Attribution Non Commercial (CC BY-NC 3.0) license, which permits others to distribute, remix, adapt, build upon this work noncommercially, and license their derivative works on different terms, provided the original work is properly cited and the use is non-commercial. See: http:// creativecommons.org/licenses/by-nc/3.0/

\section{REFERENCES}

1. Weiser TG, Regenbogen SE, Thompson KD, et al. An estimation of the global volume of surgery: a modeling strategy based on available data. Lancet 2008;372:139-44.

2. Smetana GW, Lawrence VA, Cornell JE; American College of Physicians. Preoperative pulmonary risk stratification for noncardiothoracic surgery: systematic review for the American College of Physicians. Ann Intern Med 2006;144:581-95.

3. Johnson RG, Arozullah AM, Neumayer L, et al. Multivariable predictors of postoperative respiratory failure after general and vascular surgery: results from the patient safety in surgery study. $J$ Am Coll Surg 2007;204:1188-98.

4. Finks JF, Osborne NH, Birkmeyer JD. Trends in hospital volume and operative mortality for high-risk surgery. N Engl J Med 2011;364:2128-37. 
5. Hedenstierna G, Edmark L. The effects of anesthesia and muscle paralysis on the respiratory system. Intensive Care Med 2005;31:1327-35.

6. Hedenstierna G, Edmark L. Mechanisms of atelectasis in the perioperative period. Best Pract Res Clin Anaesthesiol 2010;24:157-69.

7. Tremblay LN, Slutsky AS. Pathogenesis of ventilator-induced lung injury: trials and tribulations. Am J Physiol Lung Cell Mol Physiol 2005;288:L596-8.

8. de Prost N, Costa EL, Wellman T, et al. Effects of ventilation strategy on distribution of lung inflammatory cell activity. Crit Care 2013;17:R175.

9. Kroon AA, Delriccio V, Tseu I, et al. Mechanical ventilation-induced apoptosis in newborn rat lung is mediated via FasL/Fas pathway. Am J Physiol Lung Cell Mol Physiol 2013:305:L795-804.

10. Neto AS, Cardoso SO, Manetta JA, et al. Association between use of lung-protective ventilation with lower tidal volumes and clinical outcomes among patients without acute respiratory distress syndrome: a meta-analysis. JAMA 2012;307:2526-33.

11. Ferguson ND. Low tidal volumes for all? JAMA 2012;308:1689-90.

12. Marini JJ. Lower tidal volumes for everyone: principle or prescription? Intensive Care Med 2013;39:3-5.

13. Determann RM, Royakkers A, Wolthuis EK, et al. Ventilation with lower tidal volumes as compared with conventional tidal volumes for patients without acute lung injury: a preventive randomized controlled trial. Crit Care 2010;14:R1.

14. de Oliveira RP, Hetzel MP, Silva MA, et al. Mechanical ventilation with high tidal volume induces inflammation in patients without lung disease. Crit Care 2010;14:R39.

15. Weingarten TN, Whalen FX, Warner DO, et al. Comparison of two ventilator strategies in early patients undergoing major abdominal surgery. Br J Anaesth 2010;104:16-22.

16. Yang M, Ahn HJ, Kim K, et al. Does a protective ventilation strategy reduce the risk of pulmonary complications after lung cancer surgery?: a randomized controlled trial. Chest 2011;139:530-7.

17. Sundar S, Novack V, Jervis $\mathrm{K}$, et al. Influence of low tidal volume ventilation on time to extubation in cardiac surgical patients. Anesthesiology 2011;114:1102-10.

18. Treschan TA, Kaisers W, Schaefer MS, et al. Ventilation with low tidal volumes during upper abdominal surgery does not improve postoperative lung function. Br J Anaesth 2012;109:263-71.

19. Severgnini $P$, Selmo G, Lanza $C$, et al. Protective mechanical ventilation during general anesthesia for open abdominal surgery improves postoperative pulmonary function. Anesthesiology 2013;118:1307-21.

20. Maslow AD, Stafford TS, Davignon KR, et al. A randomized comparison of different ventilator strategies during thoracotomy for pulmonary resection. J Thorac Cardiovasc Surg 2013;146:38-44.

21. Futier E, Constantin J, Paugam-Burtz C, et al. A trial of intraoperative low-tidal-volume ventilation in abdominal surgery. N Engl J Med 2013;369:428-37.
22. Ranieri VM, Rubenfeld GD, Thompson BT, et al; ARDS Definition Task Force. Acute respiratory distress syndrome: the Berlin Definition. JAMA 2012;307:2526-33.

23. Bernard GR, Artigas A, Brigham KL, et al. The American-European Consensus Conference on ARDS. Definitions, mechanisms, relevan outcomes, and clinical trial coordination. Am J Respir Crit Care Med 1994;149(3 Pt 1):818-24.

24. Verhagen AP, de Vet HC, de Bie RA, et al. The Delphi list: a criteria list for quality assessment of randomized clinical trials for conducting systematic reviews developed by Delphi consensus. J Clin Epidemiol 1998;51:1235-41.

25. Higgins JPT, Green S, eds. Cochrane handbook for systematic reviews of interventions version 5.1.0 [updated March 2011]. The Cochrane Collaboration, 2011. http://www.cochrane-handbook.org

26. Higgins JP, Thompson SG, Deeks JJ, et al. Measuring inconsistency in meta-analyses. BMJ 2003;327:557-60.

27. Baker WL, White CM, Cappelleri JC, et al. Health Outcomes, Policy, and Economics (HOPE) Collaborative Group. Understanding heterogeneity in meta-analysis: the role of meta-regression. Int $J$ Clin Pract 2009;63:1426-34.

28. Peters JL, Sutton AJ, Jones DR, et al. Comparison of two methods to detect publication bias in meta-analysis. JAMA 2006;295:676-80.

29. Begg CB, Mazumdar M. Operating characteristics of a rank correlation test for publication bias. Biometrics 1994;50:1088-101.

30. Egger M, Davey Smith G, Schneider M, et al. Bias in meta-analysis detected by a simple, graphical test. BMJ 1997;315:629-34.

31. Zhang Z, Xu X, Ni H. Small studies may overestimate the effect sizes in critical care meta-analyses: a meta-epidemiological study. Crit Care 2013;17:R2.

32. Warn DE, Thompson SG, Spiegelhalter DJ. Bayesian random effects meta-analysis of trials with binary outcomes: methods for the absolute risk difference and relative risk scales. Stat Med 2002;21:1601-23.

33. Dellinger RP, Levy MM, Rhodes A, et al. Surviving sepsis campaign: international guidelines for management of severe sepsis and septic shock: 2012. Crit Care Med 2013;41:580-637.

34. Beecher HK. Effect of laparotomy on lung volume: demonstration of a new type of pulmonary collapse. J Clin Invest 1933;12:651-8.

35. Hammer GP, du Prel JB, Blettner M. Avoiding bias in observational studies: part 8 in a series of articles on evaluation of scientific publications. Dtsch Arztebl Int 2009;106:664-8.

36. Esteban A, Fernández-Segoviano P, Frutos-Vivar F, et al. Comparison of clinical criteria for the acute respiratory distress syndrome. Ann Intern Med 2004;141:440-5.

37. Thille AW, Esteban A, Fernández-Segoviano $\mathrm{P}$, et al. Comparison of the Berlin definition for acute respiratory distress syndrome with autopsy. Am J Respir Crit Care Med 2013;187:761-7.

38. Zhang $Z$. Protective ventilation for patients without acute respiratory distress syndrome. JAMA 2013;309:654. 